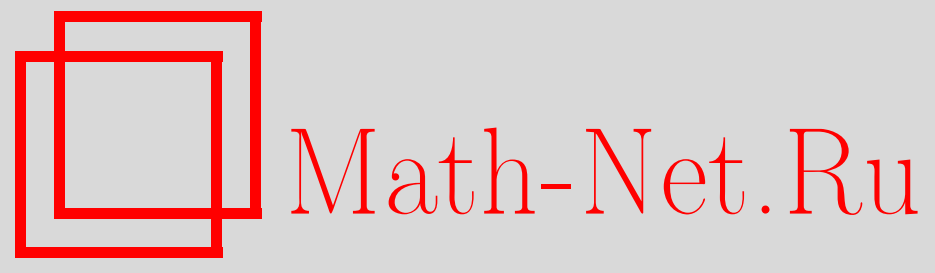

В. В. Напалков, К. Р. Зименс, Задача Валле Пуссена в ядре оператора свертки на полуплоскости, Вестн. Сам. гос. техн. ун-та. Сер. Физ.-мат. науки, 2015, номер 2, 283-292

DOI: https://doi.org/10.14498/vsgtu1355

Использование Общероссийского математического портала MathNet.Ru подразумевает, что вы прочитали и согласны с пользовательским соглашением

http://www.mathnet.ru/rus/agreement

Параметры загрузки:

IP : 54.80 .97 .219

26 апреля 2023 г., 04:05:48

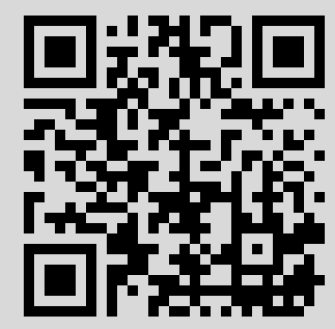




\title{
ЗАДАЧА ВАЛЛЕ ПУССЕНА В ЯДРЕ ОПЕРАТОРА СВЕРТКИ НА ПОЛУПЛОСКОСТИ*
}

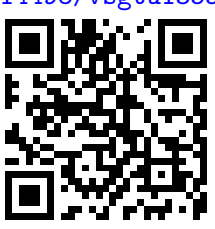

\author{
В. В. Напалков ${ }^{1}$, К. Р. Зименс ${ }^{2}$ \\ 1 Институт математики с вычислительным центром \\ Уфимского научного центра Российской академии наук, \\ Россия, 450008, Уфа, ул. Чернышевского, 112. \\ 2 Уфимский государственный авиационный технический университет, \\ Россия, 450000, Уфа, ул. К. Маркса, 12.
}

\begin{abstract}
Аннотация
Рассматривается многоточечная задача Валле Пуссена (интерполяционная задача) на полуплоскости $D, D=\{z: \operatorname{Re} z<\alpha, \alpha>0\}$. Пусть $\psi(z) \in H(D) ; \mu_{1}, \mu_{2}, \ldots \in D$ - положительные нулевые точки этой функции и их предел лежит на границе $D$. Предположим, что $\mu_{k}$ имеют кратность $s_{k}, k=1,2, \ldots$ Пусть $M_{\varphi}$ - оператор свертки с характеристической функцией $\varphi(z)$. Рассмотрим произвольную последовательность комплексных чисел $a_{k j}, j=0,1, \ldots, s_{k}-1$. Существует ли функция $u(z) \in \operatorname{Ker} M_{\varphi}$ такая, что $u^{(j)}\left(\mu_{k}\right)=a_{k j}, j=0,1, \ldots, s_{k}-1$ ? Предполагается, что характеристическая функция оператора имеет вполне регулярный рост. Получены условия разрешимости многоточечной задачи Валле Пуссена на полуплоскости. Также получены условия разрешимости поставленной задачи и на ограниченных выпуклых областях.
\end{abstract}

Ключевые слова: оператор свертки, задача Валле Пуссена, кратная интерполяция.

doi: http://dx.doi.org/10.14498/vsgtu1355

Введение. Пусть $D=\{z: \operatorname{Re} z<\alpha, \alpha>0\}, H(D)$ - пространство аналитических функций в области $D$ с топологией равномерной сходимости на компактах. Основной результат данной статьи - решение многоточечной задачи Валле Пуссена [2].

МНОГОТОЧЕЧНАЯ ЗАДАЧА ВАЛЛЕ ПУССЕНА (ИНТЕРПОЛЯЦИОННАЯ ЗАДАЧА). Пусть $\psi(z) \in H(D) ; \mu_{1}, \mu_{2}, \ldots \in D$ - положительные нулевые точки этой функции и их предел лежит на граниче $D$. Предположим, что $\mu_{k}$ имеют кратность $s_{k}, k=1,2, \ldots$. Пусть $M_{\varphi}$-оператор свертки с харак-

(C) 2015 Самарский государственный технический университет.

\section{Образец для цитирования}

Н ап ал ков В. В., З и мен с К. Р. Задача Валле Пуссена в ядре оператора свертки на полуплоскости // Вестн. Сам. гос. техн. ун-та. Сер. Физ.-мат. науки, 2015. Т. 19, № 2. C. 283-292. doi: 10.14498/vsgtu1355.

\section{Сведения об авторах}

Валентин Васильевич Напалков (д.ф.-м.н., проф., чл. корр. РАН; shaig@anrb.ru), директор института.

Карина Раисовна Зименс (karinazabirova@gmail.com; автор, ведущий переписку), аспирант, каф. специальных глав математики.

*Настоящая статья представляет собой расширенный вариант доклада [1], сделанного авторами на Четвёртой международной конференции «Математическая физика и её приложения» (Россия, Самара, 25 августа - 1 сентября 2014). 
теристической функиией $\varphi(z)$. Рассмотрим произвольную последовательность комплексных чисел $a_{k j}, j=0,1, \ldots, s_{k}-1$. Существует ли функиия $u(z) \in \operatorname{Ker} M_{\varphi}$ такая, что $u^{(j)}\left(\mu_{k}\right)=a_{k j}, j=0,1, \ldots, s_{k}-1$ ?

Ранее задача Валле Пуссена рассматривалась на всей комплексной плоскости в работах [3-8]. В работе [3] задача решается в классе дифференциальных операторов конечного порядка, в [4-6] авторы решают задачу Валле Пуссена для оператора свертки на пространстве целых функций. В статьях $[7,8]$ эта задача решается для операторов свертки Данкла и обобщенной свертки.

В настоящей работе задача Валле Пуссена [2] решается в ядре оператора свертки на полуплоскости. Берется условие, что характеристическая функция оператора имеет вполне регулярный рост [9, с. 182].

1. Предварительные сведения. Обозначим через $H^{*}(D)$ сопряженное к $H(D)$ пространство с сильной топологией. Пусть $f(z) \in H(D)$, для любого функционала $L$ из $H^{*}(D)$ существует выпуклый компакт $B_{L} \subset D$ и мера $\mu$, носитель которой лежит в $B_{L}$, такая что

$$
(L, f(z))=\int_{B_{L}} f(z) d \mu
$$

(см. [10]). Введем преобразование Лапласа

$$
\hat{L}(z)=\left(L, e^{z t}\right), \quad \hat{L}(z) \in P_{D}
$$

где пространством $P_{D}$ обозначим преобразование Лапласа всех функционалов из $H^{*}(D)$. Определим рост функции из $P_{D}$. Из интеграла $(1)$ вытекает оценка

$$
|\hat{L}(z)| \leqslant C e^{h_{L}(\theta)|z|},
$$

где $h_{L}(-\theta)$ - опорная функция множества $B_{L}$. Тогда по теореме Полиа [11, c. 51] $h_{L}(\theta)$ - индикатриса роста функции $\hat{L}(z)$. Пусть $F \in H^{*}(D) ; \varphi(z)$ - преобразование Лапласа функционала $F$. Через $D_{1}$ обозначим пересечение всех выпуклых компактов $B_{L}$ и назовем его сопряженной диаграммой функции $\varphi(z)[11$, с. 45]. Тогда $\forall \varepsilon>0$ выполняется неравенство

$$
|\varphi(z)| \leqslant C(\varepsilon) e^{\left(h_{\varphi}(\theta)+\varepsilon\right)|z|} .
$$

Определим топологию в $P_{D}$. Пусть $Q_{j}$ - последовательность выпуклых возрастающих компактов таких, что

$$
Q_{j} \subseteq Q_{j+1}^{0}
$$

где $Q_{j}^{0}$ - внутренние точки $Q_{j}$ и $\cup Q_{j}=D$. Для каждого $Q_{j}$ опорную функцию обозначим $h_{Q_{j}}(-\theta)$. Введем нормированные пространства $B_{j}$ такие, что

$$
B_{j}=\left\{\beta \in H(D):\|\beta\|_{j}=\sup _{z \in \mathbb{C}}|\beta(z)| e^{-h_{Q_{j}}(\theta)|z|}<\infty\right\}, \quad j=1,2, \ldots
$$

Тогда топология в $P_{D}$ есть индуктивный предел нормированных пространств $B_{j}$. В этой топологии последовательность $\varphi_{k} \in P_{D}$ сходится к 0 , если существуют числа $C, Q_{j 0}$ такие, что $\left|\varphi_{k}(z)\right| \leqslant C e^{h_{Q_{j 0}}(\theta)|z|}$, и $\varphi_{k}(z) \rightarrow 0$ равномерно на компактах из области $\mathbb{C}($ см. [12]). 
ОПредЕлениЕ 1 . Пусть $N$ множество в $\mathbb{C} ; q_{k} \in L-$ произвольная последовательность, где $L-$ подпространство $P_{D}$ с индуцированной из $P_{D}$ топологией. Будем говорить, что множество $N$ является секвенииалъно достаточным в $L$, если из условий

1) $\exists C>0, Q_{j}:\left|q_{k}(z)\right|<C e^{h_{Q_{j}}(\theta)|z|}, z \in N, \forall \theta, \forall k \in \mathbb{N}$,

2) $q_{k}(z) \rightarrow 0$ равномерно на любом компакте из $N$

вытекает сходимость последовательности в $L$.

Введем оператор свертки. По определению,

$$
M_{\varphi}[f(z)]=(F, f(z+t)) .
$$

Будем рассматривать такой функционал $F \in H^{*}(D)$, что $\varphi(z)=\left(F, e^{z t}\right)$. Введем условие, что функция $\varphi(z)$ - функция вполне регулярного роста вдоль вещественной оси $\left[9\right.$, с. 182]. Обозначим через $\lambda_{1}, \lambda_{2}, \ldots$ подпоследовательность нулей $\varphi(z)$ таких, что

$$
\lambda_{k} \in \mathbb{R}_{+}, \quad \lambda_{k} \nearrow \infty \quad k=1,2, \ldots
$$

Так как $\varphi(z)$ - функция вполне регулярного роста, оператор $M_{\varphi}[f(z)]$ действует из $H(D)$ в $H\left(D_{2}\right)$. Будем считать, что сопряженная диаграмма функции $\varphi(z)$ такая, что $D_{1}+D_{2}=D$. Оператор $M_{\varphi}[f(z)]$ линейный, непрерывный и сюръективный, так как $\varphi(z)$ - функция вполне регулярного роста [13].

Пусть функция $\psi$ из $H(D)$ имеет нули $\mu_{k}$ кратности $s_{k}$ такие, что

$$
\mu_{k}<\mu_{k+1}, \quad \mu_{k} \in \mathbb{R}_{+}, \quad \lim _{k \rightarrow \infty} \mu_{k}=\mu_{0}, \quad \mu_{0} \in \partial D, \quad k=1,2, \ldots
$$

Рассмотрим оператор [14] $M_{\psi}[y(z)], y(z) \in P_{D}$ :

$$
M_{\psi}[y(z)]=\frac{1}{2 \pi i} \int_{A} \psi(w) e^{z w} \gamma(w) d w
$$

где $\gamma$ - функция, ассоциированная по Борелю к $y(z)$; контур $A$ охватывает особенности $\gamma$ и не пересекает нули $\psi(z)$. Оператор действует из $P_{D}$ в $P_{D}$. Ядро оператора - множество конечных линейных комбинаций решений $z^{l} e^{\mu_{k} z}$, $0 \leqslant l<s_{k}[14]$.

Важную роль играет следующий оператор свертки $M_{\varphi}[\psi \cdot f(z)]$. Он действует линейно и непрерывно из $H(D)$ в $H\left(D_{2}\right)$. Выпишем сопряженный к нему оператор

$$
M_{\psi}[\varphi \cdot g(z)]=\frac{1}{2 \pi i} \int_{A} \psi(w) e^{z w} \gamma(w) d w
$$

где $g(z) \in P_{D_{2}} ; \gamma-$ функция, ассоциированная по Борелю к $\varphi \cdot g(z) ;$ контур $A$ охватывает особенности $\gamma$ и не пересекает нули $\psi(z)$. Этот оператор действует из $P_{D_{2}}$ в $P_{D}$ в силу того, что для произведения функций вполне регулярного роста индикаторные диаграммы складываются [9, с. 207]. 


\section{2. Решение задачи Валле Пуссена на полуплоскости.}

Теорема 1. Многоточечная задача Валле Пуссена для $M_{\varphi}$ разрешима тогда и только тогда, когда имеет место представление Фишера [3]:

$$
H(D)=\operatorname{Ker} M_{\varphi}+\{\psi(\lambda) \cdot r(\lambda): r(\lambda) \in H(D)\}
$$

где $\{\cdots\}$-множество всех произведений функиии $\psi(\lambda)$ на всевозможнье $r(\lambda) \in H(D)$.

Доказ ат ель ст в о. Решение многоточечной задачи Валле Пуссена эквивалентно тому, что для любой функции $h(z) \in H(D)$ существует решение $u(z) \in H(D)$ уравнения $M_{\varphi}[f]=0$, такое что $(u-h) / \psi \in H(D)$. Отсюда $u-h=l(z) \psi, l(z) \in H(D)$ или $h=u+l \psi$. Получили представление Фишера.

Докажем обратное. Любая функция $h(z) \in H(D)$ представима в виде

$$
h(z)=h_{1}(z)+h_{2}(z),
$$

где $h_{1}(z) \in \operatorname{Ker} M_{\varphi}, h_{2}(z) \in\{\psi(\lambda) \cdot r(\lambda): r(\lambda) \in H(D)\}$. Пусть $\mu_{k}, k=$ $=1,2, \ldots$ - нули функции $\psi$ кратности $s_{k} ; a_{k j}, j=0,1, \ldots, s_{k}-1$ - произвольная последовательность комплексных чисел. Поставим многоточечную задачу Валле Пуссена следующим образом: существует ли функция $u(z) \in \operatorname{Ker} M_{\varphi}$ такая, что $u^{(j)}\left(\mu_{k}\right)=a_{k j}$ ? Действительно, возьмем определенную функцию $h(z) \in H(D)$ такую, что $h^{(j)}\left(\mu_{k}\right)=a_{k j}$. Такая функция существует в силу теоремы Вейерштрасса. Тогда $h^{(j)}\left(\mu_{k}\right)=h_{1}^{(j)}\left(\mu_{k}\right)+h_{2}^{(j)}\left(\mu_{k}\right)$, следовательно, $a_{k j}$ есть $h_{1}^{(j)}\left(\mu_{k}\right)$. Теорема доказана.

Лемма 1. Сюргективность оператора $M_{\varphi}[\psi \cdot]$ в $H\left(D_{2}\right)$ эквивалентна представлению Фишера (6).

Лемма 1 доказывается по аналогии с [4].

Значит, из теоремы 1 и леммы 1 следует, что для решения задачи Валле Пуссена нужно доказать сюръективность оператора $M_{\varphi}[\psi \cdot]$ в $H\left(D_{2}\right)$.

Теорема 2. Пусть функиия $\psi \in H(D)$ имеет нули $\mu_{k}$ кратности $s_{k}$, которые удовлетворяют (5); функиия $\varphi(z)$ - бункиия вполне регулярного роста вдоль вещественной оси, а последовательность нулей $\lambda_{k}$ удовлетворяет (4). Тогда $N_{\varphi}=\left\{\lambda_{k}\right\}, k=1,2, \ldots$-секвенииально достаточное множество в ядре оператора $M_{\psi}$ в $P_{D}$.

Доказательст в о. В определении 1 роль $N$ будет играть $N_{\varphi}$. Нужно показать, что выполнение условий (2), (3) в точках $z \in N_{\varphi}$ влечет выполнение этих условий во всех точках плоскости $\mathbb{C}$. Рассмотрим уравнение

$$
M_{\psi}[y(z)]=0, \quad y(z) \in P_{D} .
$$

В работе [14] показано, что каждый нуль $\mu_{k}$ функции $\psi$ порождает $s_{k}$ решений уравнения (7) $e^{\mu_{k} z}, z e^{\mu_{k} z}, \ldots, z^{s_{k}-1} e^{\mu_{k} z}$ и что всякое решение уравнения (7) представляется в виде линейной комбинации этих экспонент.

Пусть задана последовательность

$$
r_{m}(z)=\sum_{k=1}^{p_{m}} \sum_{l=0}^{s_{k}-1} C_{k l}(m) z^{l} e^{\mu_{k} z}, \quad m=1,2, \ldots
$$


Предположим, что $r_{m}(z)$ сходится к нулю при $m \rightarrow \infty$ в точках $z \in N_{\varphi}$. Это означает выполнение (2) в точках $z \in N_{\varphi}$ с индикатрисой роста функции $\varphi(z)$ и выполнение (3) на любом компакте из $N_{\varphi}$.

Покажем, что в каждый член последовательности, удовлетворяющей неравенству (2) в точках $z \in N_{\varphi}$, входят только те $e^{\mu_{k} z}$, у которых $\mu_{k}<h_{Q_{j}}(\theta)$. Достаточно рассмотреть при $\theta=0$, потому что существует компакт $Q_{j 0}$, содержащий множество точек $\mu_{1}, \mu_{2}, \ldots \mu_{p}$.

Зафиксируем $m$. В последовательности $r_{m}(z)$ выберем нуль $\mu_{p}$ такой, что

$$
\mu_{p}<h_{Q_{j}}(\theta), \text { a } \mu_{p+1} \geqslant h_{Q_{j}}(\theta) \text {. }
$$

Пусть нуль $\mu_{p}$ имеет кратность $s_{p}$. Вынесем за скобки последовательности (8) элемент $z^{s_{p}-1} e^{\mu_{p} z}$. Заметим, что

$$
z^{s_{p}-1} e^{\mu_{p} z}<C e^{h_{Q_{j}}(\theta)|z|}
$$

Тогда получим

$$
z^{s_{p}-1} e^{\mu_{p} z}\left(r_{m}(z) / z^{s_{p}-1} e^{\mu_{p} z}\right) .
$$

Выражение в скобке будет стремиться к единице при $z \rightarrow \infty$ и $z \in N_{\varphi}$. Таким образом, в каждой $r_{m}(z)$ для любого $m$ последний член имеет экспоненту с показателем $\mu_{k}<h_{Q_{j}}(\theta)$. Это будет выполняться для любого $\theta$, так как опорная функция множества $Q_{j}(0)$ будет совпадать с опорной функцией множеств $Q_{j}(\theta)$ в силу того, что $\mu_{k} \in \mathbb{R}_{+}$. Значит, слагаемых в (8) конечное число.

Докажем теперь (3) для последовательности $r_{m}(z)$ на любом компакте в плоскости $\mathbb{C}$. Поскольку число членов в ряде ограничено, достаточно показать, что $C_{k l}(m) \rightarrow 0$.

Строим матрицу $A$. Берем первый элемент $e^{\mu_{1} \lambda_{1}}$, нуль $\lambda_{j 2}$ выбираем так, чтобы определитель матрицы второго порядка

$$
\Delta_{2}=\operatorname{det}\left|\begin{array}{cc}
e^{\mu_{1} \lambda_{1}} & \lambda_{1} e^{\mu_{1} \lambda_{1}} \\
e^{\mu_{1} \lambda_{j 2}} & \lambda_{j 2} e^{\mu_{1} \lambda_{j 2}}
\end{array}\right| \neq 0 .
$$

Точку $\lambda_{j 3}$ выбираем так, чтобы определитель матрицы третьего порядка

$$
\Delta_{3}=\operatorname{det}\left|\begin{array}{ccc}
e^{\mu_{1} \lambda_{1}} & \lambda_{1} e^{\mu_{1} \lambda_{1}} & \lambda_{1}^{2} e^{\mu_{1} \lambda_{1}} \\
e^{\mu_{1} \lambda_{j 2}} & \lambda_{j 2} e^{\mu_{1} \lambda_{j 2}} & \lambda_{j 2}^{2} e^{\mu_{1} \lambda_{j 2}} \\
e^{\mu_{1} \lambda_{j 3}} & \lambda_{j 3} e^{\mu_{1} \lambda_{j 3}} & \lambda_{j 3}^{2} e^{\mu_{1} \lambda_{j 3}}
\end{array}\right| \neq 0 .
$$

Аналогично строим матрицу четвертого порядка и т.д. Точку $\lambda_{j n}$ выбираем так, что $e^{\mu_{n} \lambda_{j n}}$ превосходит все элементы данной матрицы, чтобы $\Delta_{n} \neq 0$. В результате получим следующую квадратную матрицу $A$ размера $s_{1}+s_{2}+$ $+\cdots+s_{p}=d$ :

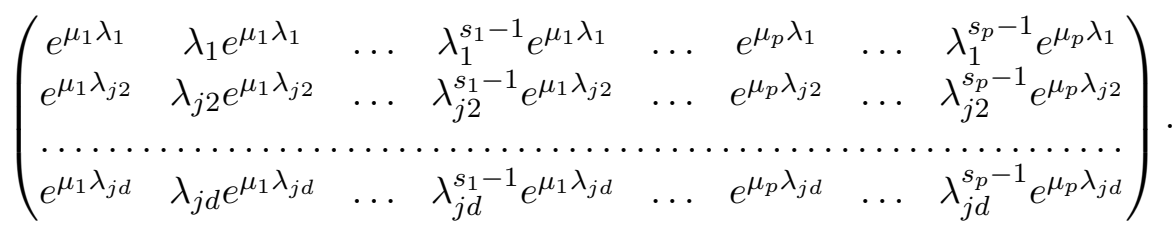


Последний элемент в главной диагонали матрицы можно сделать столь большим за счет выбора $\lambda_{j d}$ и чтобы $\operatorname{det} A \neq 0$. По формуле Крамера

$$
C_{j n}(m)=\frac{\operatorname{det}\left|\bar{A}_{j n}(m)\right|}{\operatorname{det} A},
$$

где матрица $\bar{A}_{j n}(m)$ получена из $A$ заменой $(j+n)$-ного столбца столбцом свободных членов $\left(A_{1}(m), \ldots, A_{d}(m)\right)^{\mathrm{T}}$. При этом

$$
A_{j}(m)=\sum_{k=1}^{p} \sum_{l=0}^{s_{k}-1} C_{k l}(m) \lambda_{j}^{l} e^{\mu_{k} \lambda_{j}}
$$

и $A_{j}(m) \rightarrow 0$ из $(3)$ равномерно при $m \rightarrow \infty$ на конечном множестве точек $\lambda_{j}$, следовательно, $C_{k l}(m) \rightarrow 0$ при $m \rightarrow \infty$. Поэтому для любого $z$ на компакте плоскости $\mathbb{C}$ выполняется (3) равномерно при $m \rightarrow \infty$. Теперь получаем, что $\left|r_{m}(z)\right| \rightarrow 0$ при $m \rightarrow \infty$ в плоскости $\mathbb{C}$.

Поскольку коэффициенты $C_{k l}(m)$ ограничены и $\mu_{k}<h_{Q_{j}}(\theta)$ для любого $m$ последовательности (8), получаем, что неравенство (2) будет выполняться и для точек $z$ всей плоскости $\mathbb{C}$. А это означает, что $N_{\varphi}$ является секвенциально достаточным множеством в ядре оператора $M_{\psi}$. Теорема доказана.

ЗАмечАниЕ. Из доказательства теоремы 2 вытекает, что $N_{\varphi}-$ множество единственности в ядре оператора $M_{\psi}$.

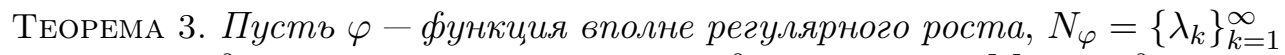
секвенииально достаточное множество в ядре оператора $M_{\psi}$, тогда оператор $M_{\varphi}[\psi \cdot]$ сюоргектиен в $H\left(D_{2}\right)$.

Доказ ательство. Сюръективность оператора $M_{\varphi}[\psi \cdot]$ - это есть замкнутость и всюду плотность его образа в $H\left(D_{2}\right)$. По теореме ДьедоннеЩварца [15] это эквивалентно инъективности оператора $M_{\psi}[\varphi \cdot]$ и замкнутости его образа в $P_{D}$.

Для линейного оператора инъективность эквивалентна тому, что его ядро тривиально. Покажем, что $\operatorname{Ker} M_{\psi}[\varphi \cdot]=\{0\}$. Пусть $g(z) \in P_{D_{2}}$ такое, что $M_{\psi}[\varphi \cdot g(z)]=0$, тогда $\varphi \cdot g(z) \in \operatorname{Ker} M_{\psi}$. Учитывая замечание к теореме 2 , получаем, что $\varphi \cdot g(z) \equiv 0$, а следовательно, $g(z) \equiv 0$, так как $\varphi(z) \neq 0$.

Для замкнутости образа оператора $M_{\psi}[\varphi \cdot]$ в $P_{D}$ необходимо показать, что если последовательность $g_{n}(z)$ сходится к $g(z)$ и $g_{n}(z) \in \operatorname{Im} M_{\psi}[\varphi \cdot]$, то $g(z) \in \operatorname{Im} M_{\psi}[\varphi \cdot]$. Так как $g_{n}(z) \in \operatorname{Im} M_{\psi}[\varphi \cdot]$, существует последовательность функций $Q_{n}(z) \in P_{D_{2}}$, удовлетворяющая следующему равенству:

$$
M_{\psi}\left[\varphi \cdot Q_{n}(z)\right]=g_{n}(z) .
$$

В работе [14] показано, что оператор $M_{\psi}$ сюръективен. Значит (см. [16]) существует непрерывный правый обратный $M_{\psi}^{-1}$, поэтому существует последовательность функций $y_{n}(z) \in P_{D}$, удовлетворяющая следующим условиям:

1) $M_{\psi}\left[y_{n}\right]=g_{n}(z)$;

2) $y_{n}(z) \rightarrow y(z), y(z) \in P_{D}$.

Из первого условия и $(9)$ в силу линейности оператора $M_{\psi}$ получим

$$
M_{\psi}\left[y_{n}(z)-\varphi \cdot Q_{n}(z)\right]=0
$$




\section{Обозначим}

$$
v_{n}(z)=y_{n}(z)-\varphi \cdot Q_{n}(z),
$$

тогда $v_{n}(z) \in P_{D}, v_{n}\left(\lambda_{k}\right)=y_{n}\left(\lambda_{k}\right)$. Так как $N_{\varphi}$ - секвенциально достаточное множество в $\operatorname{Ker} M_{\psi}$, имеет место $v_{n}(z) \rightarrow v(z)$ в $P_{D}$, где $v(z) \in \operatorname{Ker} M_{\psi}$. Тогда с учетом второго условия $\varphi \cdot Q_{n}(z)$ сходится к некоторой функции $l(z) \in P_{D}$ и нули $l(z)$ включают нули $\varphi$. Обозначим

$$
Q(z)=l(z) / \varphi
$$

Функция $l(z) \in P_{D}, \varphi(z)$ - функция вполне регулярного роста, $\varphi(z) \in P_{D_{1}}$, значит, по [9, с. 207] $Q(z) \in P_{D_{2}}$.

Покажем, что $Q_{n}(z)$ сходится к этой $Q(z)$ равномерно на компактах. Пусть $K$ - замкнутый круг с центром в нуле и $|\varphi|>\delta$ на границе $K$. Так как $\varphi \cdot Q_{n}(z)$ сходится к $l(z)$ в $P_{D}$, имеется равномерная сходимость $\varphi \cdot Q_{n}(z)$ к $l(z)$ на компактах. Это означает, что для любого $\varepsilon>0$ существует $\delta>0$ такое, что выполняется

$$
\left|\varphi \cdot Q_{n}(z)-l(z)\right|<\varepsilon, \quad n>N(\varepsilon), \quad z \in K .
$$

Следовательно, $\left|Q_{n}(z)-l(z) / \varphi\right|<\varepsilon / \delta$ на границе $K$. По принципу максимума модуля сходимость может быть продолжена на весь компакт $K$. Таким образом, $Q_{n}(z)$ равномерно сходится к $Q(z)$ на $K$. В силу сходимости $\varphi \cdot Q_{n}(z)$ к $l(z)$ в $P_{D_{1}}$ верна оценка $\left|\varphi \cdot Q_{n}(z)\right|<C e^{h_{D}(\theta)|z|}$.

Значит, согласно определению 1 получаем, что $\varphi \cdot Q_{n}(z)$ равномерно сходится к $\varphi \cdot Q(z)$ в $P_{D}$. В силу непрерывности оператора свертки имеем

$$
M_{\psi}[y(z)-\varphi \cdot Q(z)]=0
$$

Поэтому

$$
M_{\psi}[\varphi \cdot Q(z)]=M_{\psi}[y(z)]=g(z),
$$

то есть $g(z) \in \operatorname{Im} M_{\psi}[\varphi \cdot]$. Получили, что оператор $M_{\varphi}[\psi \cdot]$ сюръективен. Теорема доказана.

Таким образом, из теорем 2 и 3 следует сюръективность оператора $M_{\varphi}[\psi \cdot]$, а значит, и условия разрешения многоточечной задачи Валле Пуссена на выпуклых областях.

Благодарности. Работа выполнена при финансовой поддержке Российского фонда фундаментальных исследований (проект № 14-01-00720-а).

\section{ORCIDs}

Валентин Васильевич Напалков: http://orcid.org/0000-0001-7462-0286

Карина Раисовна Зименс: http://orcid.org/0000-0002-0340-6095

\section{БИБЛИОГРАФИЧЕСКИЙ СПИСОК}

1. Зименс К. Р., Напалков В. В. Интерполяционная задача для операторов свертки на выпуклых областях / Четвертая международная конферениия «Математическая физика и ее приложения»: материалы конф.; ред. чл.-корр. РАН И. В. Волович; д.ф.-м.н., проф. В. П. Радченко. Самара: СамГТУ, 2014. С. 181-182.

2. de La Vallée Poussin Ch. J. Sur l'equation differentielle lineaire du second ordre. Determination d'une integrale par deux valeurs assignees. Extension aux equation d'ordre $n / /$ J. Math. pures et appl., 1929. vol. 8, no. 2. pp. 125-144 (In French). 
3. Shapiro H. S. An Algebraic Theorem of E. Fischer, and the Holomorphic Goursat Problem // Bull. London Math. Soc., 1989. vol.21, no. 6. pp. 513-537. doi: 10.1112/blms/21.6.513.

4. Напалков В. В. Комплексный анализ и задача Коши для операторов свертки / Аналитические и геометрические вопросы комплексного анализа: Сборник статей. $\mathrm{K}$ 70летию со дня рождения академика Анатолия Георгиевича Витушкина / Тр. МИАН, T. 235. М.: Наука, 2001. С. 165-168.

5. Напалков В. В., Нуятов А. А. Многоточечная задача Валле Пуссена для операторов свертки // Матем. сб., 2012. Т. 203, №2. С. 77-86. doi: 10.4213/sm7763.

6. Мерзляков С. Г., Попенов С. В. Кратная интерполяция рядами экспонент в $H(\mathbb{C})$ с узлами на вещественной оси // Уфимск. матем. журн., 2013. Т. 5, № 3. С. 130-143.

7. Забирова К. Р., Напалков В. В. Операторы свёртки Данкла и многоточечная задача Валле-Пуссена // Вестн. Сам. гос. техн. ун-та. Сер. Физ.-мат. науки, 2013. № 1(30). C. 70-81. doi: 10.14498/vsgtu1139.

8. Напалков В. В., Муллабаева А. У. Об одном классе дифференциальных операторов и их применении / Тр. ИММ УрО РАН, Т. 20, 2014. С. 201-214.

9. Левин Б. Я. Распределение корней целых функций. М.: ГИТТЛ, 1956. 632 с.

10. Хермандер Л. Введение в теорию функиий нескольких комплексных переменных. М.: Мир, 1968. 280 с.

11. Леонтьев А. Ф. Целые функиии. Ряды экспонент. М.: Наука, 1989. 176 с.

12. Ткаченко В. А. Спектральная теория в пространствах аналитических функционалов для операторов, порождаемых умножением на независимую переменную // Матем. сб., 1980. Т. 112(154), № 3(7). С. 421-466.

13. В. В. Напалков Об одном классе неоднородных уравнений типа свертки // УМН, 1974. T. 29, № 3(177). C. 217-218.

14. Von Muggli H. Differentialgleichungen unendlich hoher Ordnung mit konstanten Koeffizienten// Comment. Math. Helv., 1938. vol.11, no.1. pp. 151-156. doi: 10.1007/ BF01199696.

15. Dieudonné J., Schwartz L. La dualité dans les espaces $(\mathscr{F})$ et $(\mathscr{L} \mathscr{F}) / /$ Ann. Inst. Fourier Grenoble, 1949. vol. 1. pp. 61-101 (In French).

16. Епифанов О. В. О существовании непрерывного правого обратного в одном классе локально выпуклых пространств // Изв. Сев.-Кавк. научн. иентра высш. шк., Сер. естеств. науки, 1991. №3(75). С. 3-4.

Поступила в редакцию 21/XI/2014;

в окончательном варианте - 15/II/2015;

принята в печать - 08/IV/2015. 
Vestn. Samar. Gos. Techn. Un-ta. Ser. Fiz.-mat. nauki

[J. Samara State Tech. Univ., Ser. Phys. \& Math. Sci.], 2015, vol. 19, no. 2, pp. $283-292$

ISSN: 2310-7081 (online), 1991-8615 (print)

doi: http://dx.doi.org/10.14498/vsgtu1355

MSC: 58J47, 30D05, 30E10, 46E10

\title{
DE LA VALLÉE POUSSIN PROBLEM IN THE KERNEL OF THE CONVOLUTION OPERATOR ON THE HALF-PLANE*
}

\author{
V. V. Napalkov ${ }^{1}$, K. R. Zimens ${ }^{2}$ \\ 1 Institute of Mathematics with Computing Centre, \\ Ufa Science Centre, Russian Academy of Sciences \\ 112, Chernyshevskiy st., Ufa, 450077, Russian Federation. \\ 2 Ufa State Aviation Technical University \\ 12, K. Marks st., Ufa, 450000, Russian Federation.
}

\begin{abstract}
We consider the multipoint de la Vallee Poussin (interpolational) problem in the half-plane $D, D=\{z: \operatorname{Re} z<\alpha, \alpha>0\}$. Let $\psi(z) \in H(D) ; \mu_{1}$, $\mu_{2}, \ldots \in D$ be the positive zero points of this function and let the boundary of domain $D$ contain their limit. Also, we assume that $\mu_{k}$ is of $s_{k}$ multiplicity, $k=1,2, \ldots$ Let us set $M_{\varphi}$ an operator of convolution with the characteristic function $\varphi(z)$. Taking an arbitrary sequence $a_{k j}, j=0,1, \ldots, s_{k}-1$ we should ask: is there a function $u(z) \in \operatorname{Ker} M_{\varphi}$ that provides the relation $u^{(j)}\left(\mu_{k}\right)=a_{k j}, j=0,1, \ldots, s_{k}-1$ ? We assume the operator characteristic function to be of completely regular growth. The solvability conditions for the multipoint de la Vallée Poussin problem in the half-plain and in the bounded convex domains are obtained.
\end{abstract}

Keywords: convolution operator, de la Vallée Poussin problem, multiple interpolation.

doi: http://dx.doi.org/10.14498/vsgtu1355

Acknowledgments. This work has been supported by the Russian Foundation for Basic Research (project no. 14-01-00720-a).

ORCIDs

Valentin V. Napalkov: http://orcid.org/0000-0001-7462-0286

Karina R. Zimens: http://orcid.org/0000-0002-0340-6095

(C) 2015 Samara State Technical University.

Please cite this article in press as:

Napalkov V. V., Zimens K. R. De la Vallée Poussin problem in the kernel of the convolution operator on the half-plane, Vestn. Samar. Gos. Tekhn. Univ., Ser. Fiz.-Mat. Nauki [J. Samara State Tech. Univ., Ser. Phys. \& Math. Sci.], 2015, vol. 19, no. 2, pp. 283-292. doi: 10.14498/vsgtu1355. (In Russian)

Authors Details:

Valentin V. Napalkov (Dr. Phys. \& Math. Sci., Corresponding member of RAS; shaig@anrb.ru), Director of Institute.

Karina R. Zimens (karinazabirova@gmail.com; Corresponding Author), Postgraduate Student, Dept. of Special Chapters of Mathematics.

${ }^{*}$ This paper is an extended version of the paper [1], presented at the Mathematical Physics and Its Applications 2014 Conference. 


\section{REFERENCES}

1. Zimens K. R., Napalkov V. V. The interpolation problem for convolution operators on convex domains, The 4 nd International Conference "Mathematical Physics and its Applications", Book of Abstracts and Conference Materials; eds. I. V. Volovich; V. P. Radchenko. Samara, Samara State Technical Univ., 2014, pp. 181-182 (In Russian).

2. de La Vallée Poussin Ch. J. Sur l'equation differentielle lineaire du second ordre. Determination d'une integrale par deux valeurs assignees. Extension aux equation d'ordre $n, J$. Math. pures et appl., 1929, vol. 8, no. 2, pp. 125-144 (In French).

3. Shapiro H. S. An Algebraic Theorem of E. Fischer, and the Holomorphic Goursat Problem, Bull. London Math. Soc., 1989, vol. 21, no. 6, pp. 513-537. doi: 10.1112/blms/21.6.513.

4. Napalkov V. V. Complex analysis and the Cauchy problem for convolution operators, Proc. Steklov Inst. Math., 2001, vol. 235, pp. 158-161.

5. Napalkov V. V., Nuyatov A. A. The multipoint de la Vallée-Poussin problem for a convolution operator, Sb. Math., 2012, vol.203, no.2, pp. 224-233. doi:10.1070/ SM2012v203n02ABEH004220.

6. Merzlyakov S. G., Popenov S. V. Interpolation with multiplicity by series of exponentials in $H(\mathbb{C})$ with nodes on the real axis, Ufa Math. Journal, 2013, vol. 5, no. 3, pp. 127-140. doi: $10.13108 / 2013-5-3-127$.

7. Zabirova K. R., Napalkov V. V. The Dunkl convolution operators and multipoint de la Vallée-Poussin problem, Vestn. Samar. Gos. Tekhn. Univ. Ser. Fiz.-Mat. Nauki [J. Samara State Tech. Univ., Ser. Phys. \& Math. Sci.], 2013, no. 1(30), pp. 70-81 (In Russian). doi : 10. $14498 / v s g t u 1139$.

8. Napalkov V. V., Mullabaeva A. U. On one class of differential operators and their application, Trudy Inst. Mat. i Mekh. UrO RAN, 20, 2014, pp. 201-214 (In Russian).

9. Levin B. J. Distribution of Zeros of Entire Functions. Providence, Amer. Math. Soc., 1980, $523 \mathrm{pp}$.

10. Hörmander L. An introduction to complex analysis in several variables. Princeton, D. van Nostrand Company, Inc., 1966, x+208 pp.

11. Leont'ev A. F. Tselye funktsii. Riady eksponent [Entire functions. Exponential series]. Moscow, Nauka, 1989, 176 c. (In Russian)

12. Tkachenko V. A. Spectral theory in spaces of analytic functionals for operators generated by multiplication by the independent variable, Mat. Sb. (N.S.), 1980, vol. 112(154), no. 3(7), pp. 421-466 (In Russian).

13. Napalkov V. V. A certain class of inhomogeneous equations of convolution type, Uspehi Mat. Nauk, 1974, vol. 29, no. 3(177), pp. 217-218 (In Russian).

14. Von Muggli H. Differentialgleichungen unendlich hoher Ordnung mit konstanten Koeffizienten, Comment. Math. Helv., 1938, vol.11, no.1, pp. 151-156. doi:10.1007/ BF01199696.

15. Dieudonné J., Schwartz L. La dualité dans les espaces $(\mathscr{F})$ et $(\mathscr{L} \mathscr{F})$, Ann. Inst. Fourier Grenoble, 1949, vol. 1, pp. 61-101 (In French).

16. Epifanov O. V. On the existence of the continuous right-inverse for an operator in a class of locally convex spaces, Izv. Sev.-Kavk. Nauchn. Tsentra Vyssh. Shk., Estestv. Nauki, 1991, no. 3(75), pp. 3-4 (In Russian).

Received 21/XI/2014; received in revised form $15 / \mathrm{II} / 2015$; accepted 08/IV/2015. 